\title{
İnsansız Sualtı Aracı Hareketinin Kalman Filtre ile Kestirimi ve Makine Öğrenmesi ile İyileştirilmesi
}

\author{
Estimation of Unmanned Underwater Vehicle Motion with Kalman Filter and Improvement \\ by Machine Learning
}

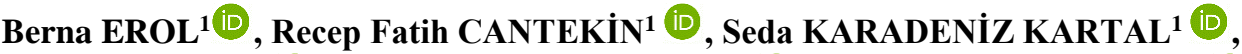 \\ Rıfat HACIOĞLU ${ }^{1} \mathbb{D}$, Kurtuluş Sedar GÖRMÜŞ² $\mathbb{D}$, Şenol Hakan KUTOĞLU² ${ }^{\mathbb{D}}$, \\ Mehmet Kemal LEBLEBícioĞLU ${ }^{3}$ iD \\ ${ }^{1}$ Zonguldak Bülent Ecevit Üniversitesi, Elektrik Elektronik Mühendisliği Bölümü, 67100, Zonguldak, Türkiye \\ ${ }^{2}$ Zonguldak Bülent Ecevit Üniversitesi, Geomatik Mühendisliği Bölümü, 67100, Zonguldak, Türkiye \\ ${ }^{3}$ Ortadoğu Teknik Üniversitesi, Elektrik Elektronik Mühendisliği Bölümü, 06800, Ankara, Türkiye
}

$\ddot{O} \mathbf{z}$

$\mathrm{Bu}$ çalı̧̧mada bir insansız sualtı aracının altı serbestlik dereceli doğrusal olmayan matematiksel modeli elde edilmiştir. Aracın matematiksel model cevabından aracın konum ve yönelim bilgileri elde edilmiştir. Elde edilen konum ve yönelim bilgilerine gürültü eklenerek navigasyon sensör verileri üretilmiştir. Üretilen gürültülü sensör verilerinin kestirimi için kokusuz ve genişletilmiş Kalman filtre algoritmaları kullanılmıştır. Kokusuz Kalman filtresinde, sistem modeli için insansız sualtı aracının doğrusal olmayan modeli kullanılmıştır. Genişletilmiş Kalman filtresinde ise sualtı aracının doğrusal olmayan modeli belirli denge noktalarında doğrusallaştırılmıştır. Kokusuz ve genişletilmiş Kalman filtresi kestirim sonuçları karşılaştırılmıştır. Kokusuz Kalman filtre ve genişletilmiş Kalman filtre kestirimlerine makine öğrenmesi olan Destek Vektör Makinesi algoritması uygulanarak, gürültünün fazla olduğu durumlar için, kestirimler iyileştirilmiştir. Buna ek olarak, aracın verilen bir kare yolu takip ettiği hareketi için kokusuz Kalman filtre ve genişletilmiş Kalman filtre kestirimleri iyileştirilmiştir. Tüm çalışma MATLAB/Simulink ortamında yapılmıştır.

Anahtar kelimeler: Genişletilmiş Kalman filtre, kokusuz Kalman filtre, destek vektör makinesi, insansız sualtı aracı, matematiksel modelleme

\begin{abstract}
In this study, the nonlinear mathematical model of unmanned underwater vehicle is obtained in 6 degrees of freedom. Position and orientation data of the vehicle are obtained from the mathematical model response of the vehicle. The navigation sensor data are generated by adding noise to the obtained position and orientation information. Extended Kalman filter and unscented Kalman filter algorithms are used to estimate noisy sensor data.. For the extended Kalman filter, nonlinear model is linearized around the equilibrium points. For the unscented Kalman filter, nonlinear system model is used. The estimation performance of extended Kalman filter and unscented Kalman filter are compared. Estimates data comes from Extended and Unscented Kalman filter are improved by applying support vector machine (SVM) which is machine learning for situations with high noise. In addition, unscented Kalman filter and extended Kalman filter estimates are improved for the given square path. All this study is modeled in MATLAB/Simulink environment.
\end{abstract}

Keywords: Extented Kalman filter, unscented Kalman filter, support vector machine, unmanned underwater vehicle, mathematical modelling

\section{GİRIŞ}

Uzun yıllardır insansız araçların takibinde doğru konum belirleme için Kalman filtresi kullanılmaktadır. Kalman filtresi konum belirleme algoritmalarının temelini oluşturmaktadır [1]. İnsansız araçların gerçek pozisyon bilgisi, nesne konumu ve hareket kontrolü için çok önemlidir [2]. Günümüzde konum takibi için en iyi yöntem olarak küresel navigasyon uydu sistemleri kullanılmaktadır [3,4]. Ancak konum belirlemede sensörler ile alınan ölçümlerde gürültü ya da dış bozucu etki olması sebebi ile ölçümlerin doğruluğu düşmektedir $[5,6]$. Kalman filtresi ile ölçümler kestirilerek daha doğru konumlandırma bilgisine ulaşılmaktadır [7]. Geleneksel Kalman filtresi (KF), sistem modeli tam olarak biliniyor ve sistem doğrusal bir sistem ise uygulanabilmektedir [8]. Doğrusal olmayan sistemlerde kestirim performansı iyi değildir [4]. Doğrusal olmayan sistemler doğrusallaştırılarak konum bilgisi kestirimi için genişletilmiş Kalman filtresi kullanılmıştır [9,10]. Genişletilmiş Kalman filtresi (EKF) doğrusal olmayan sistemler için, geçiş matrisini ve ölçüm matrisini Taylor seri açılımlarını kullanarak doğrusallaştırır [5]. EKF gerçek konum bilgisine yaklaşmaktadır. Fakat tam olarak gerçek değeri kestirememektedir. EKF'nin bu dezavantajının sebebi, doğrusal olmayan sistemlerin 
doğrusallaştırılmasında yüksek dereceli terimlerin ihmal edilerek hataya sebep olmasıdır [11]. EKF'de doğrusallaştırma yaparken matrisini hesaplar [9]. Ancak yüksek dereceli sistemlerde Jacobian matrislerin elde etmek zordur. Bu nedenle EKF' de en iyi kestirimi veremez. Doğrusal olmayan sistemlerde en iyi konum kestirimi için doğrusal olmayan sistemlere doğrusallaştırma yapılmadan uygulanabilecek kokusuz Kalman filtre (UKF) algoritması geliştirilmiştir [10].

UKF, EKF' ye nazaran rastgele bir dağılımı tahmin etmekten ise bilinen bir olasılık dağılımını tahmin etmenin daha kolay olabileceğini savunur [2]. UKF, doğrusallaştırma yapmak yerine Kokusuz Dönüşüm tekniğini kullanarak sigma noktalarını hesaplar. Buna rağmen EKF ile aynı işlem yoğunluğuna sahiptir [12]. D1ş bozucu etkisinin fazla olduğu ortamlarda UKF performansı azalabilmektedir. UKF performansını iyileştirmek için birçok farklı çalışma yapılmıştır $[13,14]$. Mevcut çalışmalardan farklı olarak KF çeşitlerinin kestirimlerini iyileştirmek için günümüzde popüler olan makine öğrenmesi algoritmaları kullanılabilir [15]. Makine öğrenme algoritmaları tahmin problemlerinde kullanılmaktadır. Makine öğrenmesi, oluşturulan veri setindeki giriş ve çıkış değerlerine göre tahmin yapabilen bilgisayar algoritmalarının genel adıdır [16]. Birçok makine öğrenmesi algoritması vardır. Bunlardan biri Destek Vektör Makinesi (SVM)'dir. SVM, Vapnik ve ark. tarafından 1995'te geliştirilmiştir [17]. SVM algoritması hem siniflandirma hem de regresyon problemlerini çözmek için kullanılan güçlü bir makine öğrenmesi algoritmasıdır [18]. SVM şimdiye kadar pillerin kalan ömrünü tahmin etme, enflasyon tahmini, konut fiyat tahmini gibi birçok farklı alanlarda kullanılmıştır [19-22]. Genel olarak sınıflandırma problemlerinde kullanılan SVM, regresyon için kullanılması Smola ve ark. tarafından ileri sürülmüştür [23]. Bu yöntem Destek Vektör Regresyonu (SVR) olarak adlandırılmaktadır. SVR algoritması, elektrik yük tahmini [24], gemi hareketini tahmin etmek için [25], filtre kestiriminin iyileştirilmesi [15] ve günümüzde birçok alanda kullanılmaktadır [26,27].

$\mathrm{Bu}$ çalışmada insansız sualtı aracının navigasyon sensör verisini, simülasyon bazlı üretmek için, aracın 6 serbestlik dereceli doğrusal olmayan matematiksel modeli kullanılmıştır. Elde edilen sensör verilerine sıfır ortalamalı Gauss gürültüsü eklenmiş ve KF çeşitleri uygulanarak durumlar kestirilmiştir. Doğrusallaştırılmış araç modeline EKF, doğrusal olmayan modele ise UKF uygulanmıştır. UKF ve EKF kestirim performansları karşılaştırılmıştır. Çok gürültülü (dış bozucunun fazla olduğu ortamlarda) sensör verilerinde de filtre performanslar1 incelenmiştir. Çok gürültülü sensör verilerinde UKF ve EKF kestirimlerinin performans1 kötüleşebilmektedir. Gürültülü verilerde daha doğru konum kestirimi için UKF ve EKF kestirimlerine makine öğrenmesi SVM algoritması uygulanmıştır. Uygulanan algoritma sonrasinda EKF kestirimlerinin değişiklik gösterdiği, UKF kestirimlerinin ise iyileştiği gözlemlenmiştir.

\section{MATEMATIKSEL MODELLEME VE SENSÖR VERISII OLUŞTURMA}

Bir insansız sualtı aracının doğrusal olmayan matematiksel modeli Eşitlik (1) ve Eşitlik (2) de tanımlanmıştır [28].

$M(\dot{v})+C(v) v+D(v) v+g(\eta)=\tau=u$

$\dot{\eta}=J(\eta) v$

$M$ : Aracın kütle matrisi,

$C$ : Merkezkaç kuvveti ve Coriolis kuvveti matrisi,

$D$ : Sönümleme matrisi,

$g$ : Yerçekimi ve suyun kaldırma kuvveti matrisi,

$\tau$ : Girdi vektörü,

$v$ : Aracın doğrusal $\left(v_{1}\right)$ ve açısal hız $\left(v_{2}\right)$ vektörü,

$\eta$ : Aracin doğrusal $\left(\eta_{1}\right)$ ve açısal konum $\left(\eta_{2}\right)$ (yönelim) vektörü,

$J$ : Koordinat çevirim matrisi.

İnsansız sualtı aracının matematiksel modeli için, aracın 6 serbestlik dereceli (DOF) doğrusal olmayan modeli için kullanılan vektörler Eşitlik (3), (4) ve (5)'te verilmiştir [28].

$$
\begin{aligned}
& \vec{\eta}=\left[\eta_{1}^{T}, \eta_{2}^{T}\right]^{T} \quad \eta_{1}^{T}=[x, y, z]^{T} \quad \eta_{2}^{T}=[\emptyset, \theta, \psi]^{T} \\
& \vec{v}=\left[v_{1}^{T}, v_{2}^{T}\right]^{T} \quad \overrightarrow{v_{1}}=[u, v, w]^{T} \quad \overrightarrow{v_{2}}=[p, q, r]^{T} \\
& \vec{\tau}=u=\left[\tau_{1}^{T}, \tau_{2}^{T}\right]^{T} \quad \overrightarrow{\tau_{1}}=[X, Y, Z]^{T} \quad \overrightarrow{\tau_{2}}=[K, M, N]^{T}
\end{aligned}
$$

İticilerin ürettikleri moment ve kuvvetlerden oluşan $\mathrm{u}$ matrisi sistem girdisidir. Şekil 1'de bu çalışmada kullanılan insansız sualtı aracı verilmiştir. Şekil 1'de gösterilen araçta $\mathrm{x}$ ekseni yönündeki (yatay konum) hareketi ve $\mathrm{z}$ ekseni etrafındaki dönmeyi (sapma açıs1) sağlayan 2 tane yatay konumda itici bulunmaktadır, y ekseni etrafındaki dönmeyi sağlayan 1 tane dikey konumda itici bulunmaktadır [29].

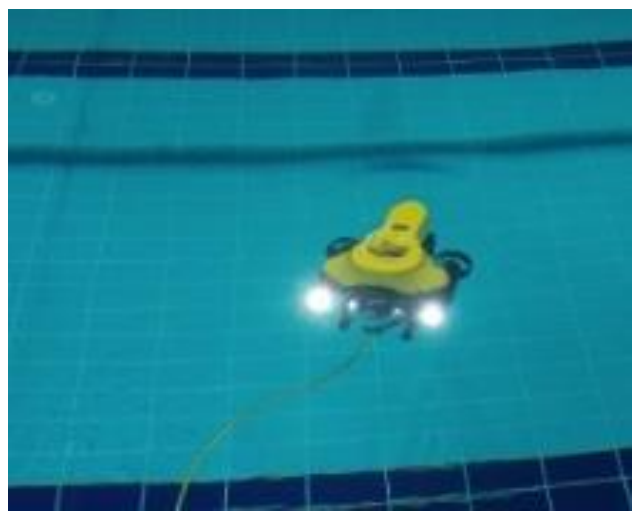

Şekil 1. Bu çalışmada kullanılan insansız sualtı aracı (Zonguldak Bülent Ecevit Üniversitesi havuz deneyinden) 
$\mathrm{Bu}$ çalışmada, insansız sualtı aracının $\mathrm{x}$ ve $\mathrm{y}$ eksenlerindeki konum bilgisi akustik bazlı konum ölçüm sistemi olan çok kısa taban hatlı, (Ultra short base line) USBL'den (GPS entegreli), yönelim bilgisi ataletsel ölçüm sistemi, (Inertial Measurement Unit) IMU' dan alındığı, aracın z eksenindeki konumu araca entegre edilen basınç sensöründen de alındığı düşünülmüştür. Su üstü aracına USBL/GPS in GPS modülü yerleştirilerek USBL'in 4 adet alıcı antenleri, yerleri bilinen su üstü aracının 4 köşesine yerleştirilmiştir (P1, P2, P3, P4).

USBL'in alıcı vericisi insansız sualtı aracına entegre edilmiştir. İnsansız sualtı aracındaki alıc1-vericiden gönderilen akustik ses sinyali su üstü aracındaki 4 adet alıcı tarafindan cevaplanarak tekrar insansız sualtı aracındaki alıcı-verici tarafından alınmıştır. Böylelikle ses sinyal hızı ve sinyalin seyahat süresinden her bir anten ile insansız sualtı aracı arasındaki mesafeler elde edilmektedir. Yine bu mesafeler aynı zamanda SLAM, eş zamanlı konum belirleme algoritmasından elde edilebilmektedir. Böylelikle d1, d2, d3, d4 mesafelerinin aynı zamanda akustik sinyalin hız ve seyahat süresinin çarpımına eşit olduğu düşünülerek insansız sualtı aracinın bilinmeyen konumu $\mathrm{P}(\mathrm{x}, \mathrm{y}, \mathrm{z})$ elde edilir.

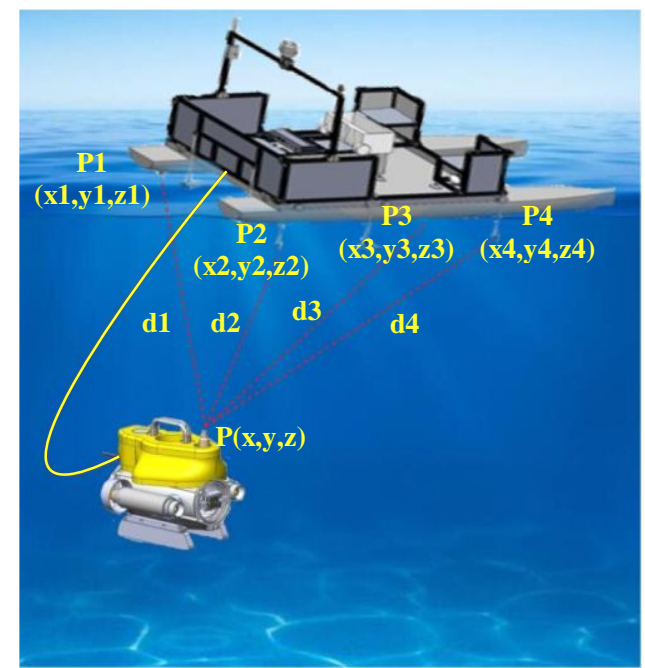

Şekil 2. İnsansız sualtı aracı navigasyonu için tasarlanan sistemin şematik gösterimi

İnsansız sualtı aracına yerleştirilen verici-alıcıdan giden sinyal su üstündeki alıcılar tarafindan cevaplanarak tekrar sualtı aracına ulaşmaktadır. $\mathrm{Bu}$ geçen süreden ve ses hızından faydalanılarak iki araç arasındaki mesafe ve yönelim ölçülür. Aracın konum bilgisinin elde edilmesi için en az 3 tane su üstü alıcısına ihtiyaç vardır. $\mathrm{Bu}$ ölçülen bilgilerden faydalanılarak su altındaki aracın konumu hesap edilir. $\mathrm{Bu}$ çalışmada aracın $\mathrm{x}$ eksenindeki konumu ile $\mathrm{z}$ ekseni etrafındaki dönüş açısını oluşturmak için insansız sualtı aracinın matematiksel modeli kullanılmıştır. Modelden elde edilen gerçek konum ve açı bilgilerine sıfır ortalamalı gauss gürültüsü eklenerek sensör verileri üretilmiştir.

\section{SENSÖR VERILLERININ KESTIRIMI}

$\mathrm{Bu}$ çalışmada insansız sualtı aracının model cevabından üretilen gürültülü sensör verilerini kestirmek için UKF ve EKF algoritmaları kullanılmıştır. İnsansız sualtı aracının doğrusal olmayan modeli için UKF ve doğrusallaştırılmış modeli için EKF algoritmaları kullanılmıştır. Gürültünün fazla olduğu durumda, makine öğrenme algoritması, KF algoritmalarına entegre edilerek iyileştirilmiştir.

\subsection{Genişletilmiş Kalman Filtresi}

Geleneksel KF doğrusal olmayan sistemlere uygulanamamaktadır. Doğrusal olmayan sistemlere KF uygulayabilmek için doğrusallaştırma yapmak gerekir [10]. KF'nin doğrusal olmayan sistemlere uygulanabildiği ilerletilmiş formuna "Genişletilmiş Kalman Filtresi (EKF)" denir. Doğrusallaştırma yapmanın birçok metodu vardır [30]. Bunlardan bir tanesi diferansiyelleme metodudur. $\mathrm{Bu}$ çalışmada, EKF için sistem modeli olarak, insansız sualtı aracının doğrusal olmayan modeli belirli denge noktalarında doğrusallaştırılarak kullanılmıştır.

\subsubsection{Insansız sualtı aracının doğrusal hareket denklemleri}

İnsansız sualtı aracının doğrusal hareket denklemleri, Eşitlik (1) ve Eşitlik (2) de belirtilen doğrusal olmayan hareket denklemlerinin zamana göre değişen bir referans yörünge veya denge noktası etrafinda doğrusallaştırılmasıyla elde edilir. Burada referans noktaları $\eta_{0}$ (konum için) ve $v_{0}$ (hız için) olarak alınırsa Eşitlik (1) diferansiyellenerek, aşağıdaki gibi doğrusallaştırılır [28]. Aynı zamanda doğrusallaştırma yapılabilmesi için aracın sabit hızda bir çalışma noktası seçilip, bu noktada yerçekimi kuvveti ve suyun kaldırma kuvveti terimleri ihmal edilir.

$\Delta_{v}=v-v_{0}=x_{1} \quad$ (referans noktasindan sapmay 1 gösterir)

$\Delta_{\eta}=\eta-\eta_{0}=x_{2} \quad$ (referans noktasindan sapmay gösterir)

$M \Delta_{\dot{v}}+\left.\frac{\partial C(v) v}{\partial v}\right|_{v_{0}} \Delta_{v}+\left.\frac{\partial D(v) v}{\partial v}\right|_{v_{0}} \Delta_{v}+\left.\frac{\partial g(\eta)}{\partial \eta}\right|_{\eta_{0}} \Delta_{\eta}=\Delta_{\tau}$

Zamana bağımlı doğrusallaştırılmış dinamik hareket denklemi Eşitlik (9) da verildiği gibidir.

$M \dot{x}_{1}+C(t) x_{1}+D(t) x_{1}+G(t) x_{2}=\tau$

Burada; $C(t)=\left.\frac{\partial C(v) v}{\partial v}\right|_{v_{0(t)}}, D(t)=\left.\frac{\partial D(v) v}{\partial v}\right|_{v_{0}(t)}$ ve $G(t)=$ $\frac{\partial g(\eta)}{\partial \eta} \int_{\eta_{O(t)}}$ şeklindedir. 
Eşitlik (2) aşağıdaki gibi doğrusallaştırılır:

$\Delta_{\dot{\eta}}=J\left(\eta_{0}\right) \Delta_{v}+J^{*}\left(v_{0}, \eta_{0}\right) \Delta_{\eta}$

$J^{*}\left(v_{0}, \eta_{0}\right) \Delta_{\eta}=\left[J\left(\eta_{0}+\Delta_{\eta}\right)-J\left(\eta_{0}\right)\right] v_{0}$

Zamana bağımlı doğrusallaştırılmış kinematik hareket denklemi aşağıdaki gibidir:

$\dot{X}_{2}=J(t) x_{1}+J^{*}(t) x_{2}$

Burada; $J(t)=J\left(\eta_{0}(t)\right)$ ve $J^{*}(t)=J^{*}\left(v_{0}(t), \eta_{0}(t)\right)$ şeklindedir. $J^{*}=0$ alınarak zamandan bağımsız model Eşitlik (13) da, $\dot{x}=A x+B u$ formunda olur.

$\left|\begin{array}{c}\dot{x}_{1} \\ \dot{x}_{2}\end{array}\right|=\left|\begin{array}{cc}-M^{-1}[C+D] & -M^{-1} G \\ J & 0\end{array}\right| \cdot\left|\begin{array}{c}x_{1} \\ x_{2}\end{array}\right|+\left|\begin{array}{c}-M^{-1} \\ 0\end{array}\right| \cdot u$

EKF tasarımında Jacobian matrisleri hesaplanır. $\mathrm{Bu}$ adım, ölçüm matrisi $(H)$ ve geçiş matrisinin $(F)$ diferansiyellenmesiyle doğrusallaştırılmasıdır [30].

$H_{k}=\left.\frac{\partial h\left(x_{k}, k\right)}{\partial x}\right|_{x=\hat{x}_{k}^{-}}$

$F(k+1, k)=\left.\frac{\partial f(x, k)}{\partial x}\right|_{x=\hat{x}_{k}}$

EKF uygulamasinda, insansiz sualtı aracı hareket denklemlerinin zamandan bağımsız formları dikkate alınmıştır. Durum tahmini ayrık zaman geçiş matrisi ile Eşitlik (16) daki gibi ilişkilendirilir.

$x_{k+1}=\phi_{k} \cdot x_{k}$

KF'nin ayrık zaman formu Eşitlik (17) de sunulmuştur [1].

$\phi_{k}=e^{F \Delta t}=I+F \Delta t+\frac{(F \Delta t)^{2}}{2}+\frac{(F \Delta t)^{3}}{6}+\ldots$

Burada:

$\Delta t=t_{k+1}-t_{k}$

Zaman aralığ1 olarak belirlenir [1] Doğrusallaştırmada ikinci derece terimler ihmal edilmektedir [28]. Bu yüzden Eşitlik (17) için ikinci ve daha yüksek dereceli terimler ihmal edilir.

\subsubsection{Genişletilmiş Kalman filtresi algoritması}

Sistem modeli için Eşitlik (13) ve Eşitlik (15) kullanılmıştır. Durum vektörü x Eşitlik (19) daki gibidir.

$$
x=/ \eta_{1} \quad \eta_{2} \quad v_{1} \quad v_{2} / T
$$

Q matrisi, sistem gürültü kovaryans matrisidir ve köşegen matristir [1]. Bu çalışmada $12 \times 12^{\prime}$ 'lik köşegen matris alınmıştır. İnsansız sualtı aracı sistem gürültü kaynakları; insansız sualtı aracının matematiksel modelinden kaynaklı konumda ve hızda meydana gelen rastgele hatalardır. Her bir terim sistem durumlarının standart sapmaları ile orantılıdır.

$Q=\left|\begin{array}{cccc}Q_{11} & 0 & \ldots & 0 \\ 0 & Q_{22} & 0 & \vdots \\ \vdots & 0 & \ddots & 0 \\ 0 & \ldots & 0 & Q_{1212}\end{array}\right|$

Burada;

- $\quad Q_{11}, Q_{22}$ ve $Q_{33}$ sirasiyla x, y ve z yönündeki hareketin sistem gürültüsüdür

- $Q_{44}, Q_{55}$ ve $Q_{66}$ sirasiyla x, y ve z ekseni etrafındaki dönme için sistem gürültüsüdür.

- $\quad Q_{77}, Q_{88}$ ve $Q_{99}$ sirasiyla x, y ve z yönündeki hareket için doğrusal hız sistem gürültüsüdür.

- $\quad Q_{1010}, Q_{1111}$ ve $Q_{1212}$ sirasiyla x, y ve z ekseni etrafındaki dönme için açısal hız sistem gürültüsüdür.

Ölçüm modeli için z ölçüm vektörü Eşitlik (21) ve $H$ ölçüm matrisi Eşitlik (23) daki gibi tanımlanmıştır.

$z_{k}=H_{k} \cdot x_{k}$

$z=\mid$ konum yönelim $\left.\right|^{T}$

$H$ matrisi ölçüm matrisidir ve birim matristir [1]. Bu çalışmada $6 \times 12$ 'lik birim matris alınmıştır.

$H=\left|\begin{array}{llll}I_{3} & O_{3} & O_{3} & O_{3} \\ O_{3} & I_{3} & O_{3} & O_{3}\end{array}\right|$

$R$ matrisi ölçüm gürültü kovaryansıdır ve köşegen matristir. $\mathrm{Bu}$ çalışmada $6 \times 6$ 'lık köşegen matris alınmıştır. $R$ matrisindeki köşegen terimleri, her bir ölçümün varyanslarıdır. İnsansız sualtı aracı ölçüm gürültü kaynakları; USBL/GPS kaynaklı konumdaki rastgele hatalar ve yönelim ölçümünde meydana gelen rastgele hız hatalarıdır.

$R=\left|\begin{array}{llllll}R_{11} & 0 & 0 & 0 & 0 & 0 \\ 0 & R_{22} & 0 & 0 & 0 & 0 \\ 0 & 0 & R_{33} & 0 & 0 & 0 \\ 0 & 0 & 0 & R_{44} & 0 & 0 \\ 0 & 0 & 0 & 0 & R_{55} & 0 \\ 0 & 0 & 0 & 0 & 0 & R_{66}\end{array}\right|$

Burada;

- $\quad R_{11}, R_{22}$ ve $R_{33}$ sirasiyla $\mathrm{x}, \mathrm{y}$ ve $\mathrm{z}$ yönündeki hareketin ölçüm gürültüsüdür.

- $\quad R_{44}, R_{55}$ ve $R_{66}$ sirasiyla $\mathrm{x}, \mathrm{y}$ ve $\mathrm{z}$ ekseni etrafındaki dönme için ölçüm gürültüsüdür. 
EKF algoritması da geleneksel KF algoritma adımlarına göre işler. EKF algoritmasındaki fark, sistem modeli için yukarıda belirtilen diferansiyelleme ile doğrusallaştırma yapılması ve Jacobian matrislerinin türetilmesidir. EKF algoritması aşağıda sunulduğu gibidir. $\mathrm{Bu}$ algoritmada 1. ve 2. adım zaman güncelleme (sistem yayılımı) aşamasıdır. Sonraki adımlar ölçüm güncelleme aşamasıdır.

1. Durum tahmini hesapla.

$\hat{x}_{k+1}^{-}=\phi \cdot \hat{x}_{k}^{+}$

2. Hata kovaryansı hesapla.

$P_{k+1}^{-}=\phi_{k} \cdot P_{k} \cdot \phi_{k}^{T}+Q_{k}$

3. Kalman kazancını hesapla.

$K_{k}=P_{k}^{-} \cdot H_{k}^{T} \cdot\left(H_{k} \cdot P_{k}^{-} \cdot H_{k}^{T}+R_{k}\right)^{-1}$

4. Durum tahminini güncelle.

$\hat{x}_{k+1}^{+}=\hat{x}_{k+1}^{-}+K_{k} \cdot\left[z_{k}-h \cdot\left(\hat{x}_{k+1}^{-}, t_{k}\right)\right]$

5. Hata kovaryansını güncelle.

$P_{k+1}^{+}=\left(I-K_{k} \cdot H_{k}\right) \cdot P_{k+1}^{-}$

\subsection{Kokusuz Kalman Filtresi}

EKF, doğrusal olmayan sistemlere uygulanabilir. Ancak doğrusallaştırma yapmak iki dezavantaja neden olur [28]. Bunlardan birisi yüksek dereceli terimlerin ihmal edilmesidir. Bunun sonucunda gerçek değerden istenilmeyen derecede uzaklaşan filtreler oluşabilir. İkincisi, Jacobian matrislerinin bazı uygulamalarda türetilmesine gerek yoktur. Ancak her uygulama için Jacobian matrislerini türetmek uygulamada önemli zorluklara yol açar [8]. EKF' nin bu dezavantajlarını, UKF ortadan kaldırmıştır. UKF' nin çalışma prensibi, rastgele bir olasılık dağılımını tahmin etmektense bilinen bir olasılık dağılımını (Gaussian) tahmin etmenin daha kolay olduğudur [31]. UKF, doğrusal olmayan sistemlere doğrudan uygulanabilmektedir. UKF, kokusuz dönüşüm metodu kullanılarak uygulanır. Kokusuz dönüşüm doğrusal olamayan bir dönüşüm geçiren rastgele değişkenin ortalama ve kovaryansları hesaplamak için kullanılır [32]. Birçok kokusuz dönüşüm tekniği vardır. $\mathrm{Bu}$ çalışmada, karekök kovaryans tipi kullanılmıştır. UKF' deki ikinci önemli husus ise sigma noktalarıdır. Sigma noktaları UKF kestiriminde bir önceki ölçümün istatistiğinden elde edilen aracın olabileceği muhtemel konumlardır. Örnek olarak, sigma noktaları Şekil 3'de gösterilmektedir.

Sigma noktalarından oluşan $S$ matrisi $2 n+1$ boyutundadir. Burada $n$ durum matrisinin boyutudur. Sigma noktalarının ortalamasının ağırlığ $w_{m}$ ve kovaryansının ağırlığı $w_{c}{ }^{\prime}$ dir. Ayrıca sigma noktalarının ortalaması durum vektör tahminini, varyans1 ise hata kovaryans matrisini ifade eder [1]. Sigma noktaları Eşitlik (30), Eşitlik (31) ve Eşitlik (32) gibi elde edilir:

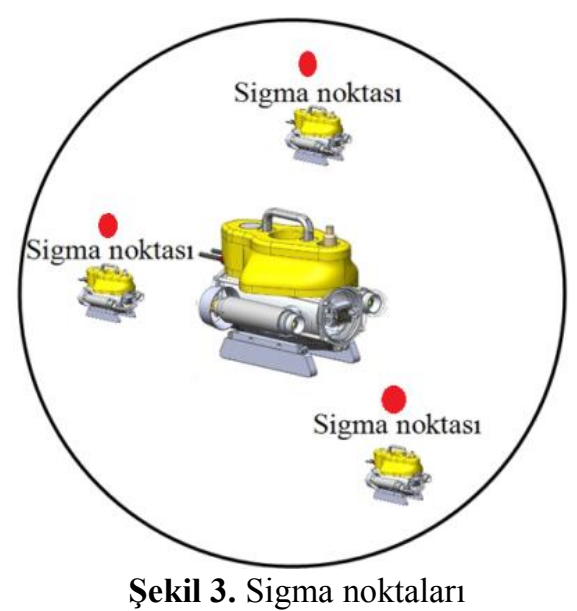

$S_{0}=\bar{x}, i=0$

$S_{i}=\bar{x}+\sqrt{\left(n+K_{-} \operatorname{sig}\right) \cdot P_{k-1}}, i=1,2, \ldots, n$

$S_{i}=\bar{x}-\sqrt{\left(n+K_{-} \operatorname{sig}\right) \cdot P_{k-1}}, i=n+1, \ldots, 2 n$

Kokusuz dönüşümde, ölçekleme parametreleri bulunur ve bu ölçekleme paremetlerinin modele uygun bir şekilde belirlenmesi filtre performansını artırır. $K_{-}$sig, ölçekleme parametresi, sigma noktasının orijine konumlandırılmış $x$ rastgele değişkeninden ne kadar uzaklaşabileceğini kontrol eder [2].

$K \_$sig $=\alpha^{2} .(n+\lambda)-n$

Burada, $\alpha$, birincil ölçekleme parametresidir. Sigma noktalarının $\bar{x}$ etrafindaki yayılımını belirler. Genellikle $\quad 10^{-4} \leq \alpha \leq 1$ şeklinde küçük ve pozitif bir sayı seçilmelidir. Çünkü istenilen durum, $\bar{x}$ değerinden uzaklaşmamaktır. $\lambda$, üçüncül ölçeklendirme parametresidir. Genellikle 0 veya 3-n şeklinde belirlenir [32].

$w_{m}=w_{c}=\frac{1}{2 \cdot\left(n+K_{-} \text {sig }\right)}$

$w_{0}^{c}=\frac{K_{-} \operatorname{sig}}{\left(K_{-} \operatorname{sig}+n\right)}+\left(1-\alpha^{2}+\beta\right)$

$w_{0}^{m}=\frac{K_{-} s i g}{\left(K_{-} \operatorname{sig}+n\right)}$

Burada, $\beta$, İkincil ölçeklendirme parametresidir ve $x$, in önceki dağılımlarının bilgisini dahil etmek için kullanılır ve Gauss dağılımları için en uygun 2 alınır [32]. UKF'deki ilk adım hata kovaryans matrisinin $(P)$ karekökünün alınmasıdır. Ardından sigma noktaları, hata kovaryansının karakökü ve ölçeklendirme parametreleri doğrultusunda hesaplanır. Daha sonra 
durum kestirimi hesaplanmasında kullanılarak algoritma işleyişi KF ve EKF'deki gibi devam eder.

\subsubsection{Kokusuz Kalman filtresi algoritması}

UKF tasarımında, insansız sualtı aracının Eşitlik (1) ve Eşitlik (2) deki doğrusal olmayan hareket denklemlerinden yola çıkılarak aşağıdaki doğrusal olmayan durum uzay modeli ede edilir. Durum seçimi ve ölçüm modeli EKF'deki gibidir.

$\left|\begin{array}{l}\dot{x}_{1} \\ \dot{x}_{2}\end{array}\right|=\left|\begin{array}{cc}-M^{-1}[C(v)+D(v)] & 0 \\ J(\eta) & 0\end{array}\right| \cdot\left|\begin{array}{c}x_{1} \\ x_{2}\end{array}\right|+\left|\begin{array}{c}-M^{-1} \\ 0\end{array}\right| \cdot u+\left|\begin{array}{c}-g(\eta) \\ 0\end{array}\right|$

Doğrusal olmayan durum uzayı gösterimi aşağıdaki gibi olur:

$\dot{x}=A(x) x+B(x) u+f(x)$

Burada; $f=\left|\begin{array}{c}0 \\ -g(\eta)\end{array}\right|$ olur. UKF algoritması aşağıda sunulduğu gibidir. $\mathrm{Bu}$ algoritmada 3. 4. ve 5. adım zaman güncelleme (sistem yayılımı) aşamasıdır. Sonraki adımlar ölçüm güncelleme aşamasıdır.

1. Başlangıç değerlerini belirle.

$\hat{x}_{0}=E\left[x_{0}\right]$

$P_{0}=E\left[\left(x_{0}-\hat{x}_{0}\right)\left(x_{0}-\hat{x}_{0}\right)^{T}\right]$

2. Sigma noktalarını belirle.

$S_{k-1}=\left[\hat{x}_{k-1}^{-}, \hat{x}_{k-1}^{-}+\sqrt{\left(n+K_{-} s i g\right) \cdot P_{k-1},}, \hat{x}_{k-1}^{-}-\sqrt{\left.\left(n+K_{-} s i g\right) \cdot P_{k-1}\right]}\right.$

3. Durum tahmini hesapla.

$\hat{x}_{k+1}^{*}=\phi_{k} \cdot S_{k-1}$

$\hat{x}_{k}^{-}=\sum_{i=0}^{2 n} w_{i}^{(m)} . \hat{x}_{i, k+1}^{*}$

4. Hata kovaryansını hesapla.

$P_{k}^{-}=\sum_{i=0}^{2 n} w_{i}^{(c)} \cdot\left(\hat{x}_{i, k+1}^{*}-\hat{x}_{k}^{-}\right)\left(\hat{x}_{i, k+1}^{*}-\hat{x}_{k}^{-}\right)^{T}+Q$

5. Ölçümü formüle et.

$\hat{y}_{k-1}=H \cdot\left(\hat{x}_{k+1}^{*}\right)$

$\hat{y}_{k}=\sum_{i=0}^{2 n} w_{i}^{(m)} \cdot \hat{y}_{i, k-1}$

6. Kalman kazancını hesapla.
$P_{y y}=\sum_{i=0}^{2 n} w_{i}^{(c)} \cdot\left(\hat{y}_{i, k-1}-\hat{y}_{k}\right)\left(\hat{y}_{i, k-1}-\widehat{y}_{k}\right)^{T}+R$

$P_{x y}=\sum_{i=0}^{2 n} w_{i}^{(c)} \cdot\left(\hat{x}_{i, k+1}^{*}-\hat{x}_{k}^{-}\right)\left(\hat{y}_{i, k-1}-\widehat{y}_{k}\right)^{T}$

$K_{k}=P_{x y} \cdot P_{y y}^{-1}$

7. Durum tahminini güncelle.

$\hat{x}_{k}^{+}=\hat{x}_{k}^{-}+K_{k} \cdot\left(z-\hat{y}_{k}\right)$

8. Hata kovaryansını güncelle.

$P_{k}^{+}=P_{k}^{-}-\left(K_{k} \cdot P_{y y} \cdot K_{k}^{T}\right)$

\subsection{Destek Vektör Makinesi}

Destek vektör makinesi (SVM), tahmin ve sınıflandırma problemlerinde kullanılan popüler bir makine öğrenmesi algoritmasıdır [33]. Sınıflandırma problemlerinde daha sik kullanılan SVM, regresyon problemlerinde kullanılması Smola ve ark. tarafından önerilmiştir [23]. SVM, Şekil 4'teki gibi giriş-çıkış ilişkisi doğrusal ve doğrusal olmayan problemlerde kullanılabilir.

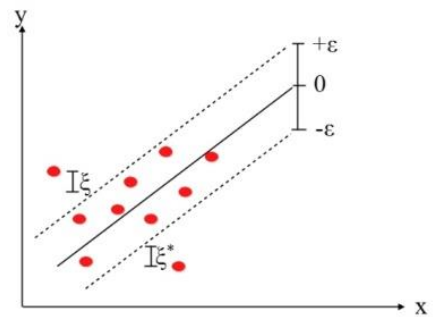

a) Doğrusal SVM
Şekil 4. Tek boyutlu SVM

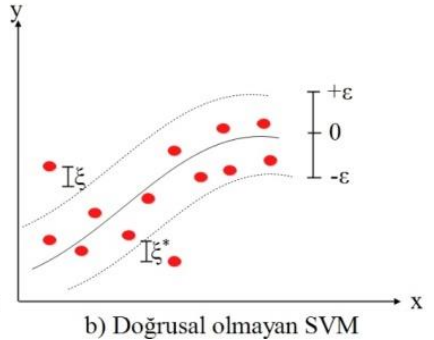

b) Doğrusal olmayan SVM
$D=\{(x 1, y 1),(x 2, y 2), \ldots,(x i, y i)\}$ den oluşan bir eğitim seti olsun. Burada $x i$ giriş değişkenleri, $y i$ ise bu girişlere denk gelen çıkış değişkenleridir.

SVM'de amaç verilen giriş değişkenleri ile çıkış değişkenleri arasındaki bağlantıyı kurmaktır. Yani SVM algoritması $x i$ 'ler ile $y i$ 'lerin ilişkisini en doğru şekilde oluşturulacak fonksiyonu bulmaktadır. SVM bu bağlantıyı kurarken Eşitlik (52) da verilen J maliyet fonksiyonunu minimize eden optimizasyon problemini çözmektedir.

Maliyet fonksiyonu ( $\mathrm{J})$ :

$J=\frac{1}{2} / / w / \|^{2}+C \cdot \sum_{i=1}^{m}\left(\xi_{i}+\xi_{i}^{*}\right)$

Kisitlar: 
$y_{i}-\left(w \cdot x_{i}\right)-b \leq \varepsilon+\xi_{i}$

$\left(w \cdot x_{i}\right)+b-y_{i} \leq \varepsilon+\xi_{i}^{*}$

$\xi_{i}, \xi_{i}^{*} \geq 0, \quad i=1, \ldots, m$

Burada, $C$ parametresi sıfirdan büyük bir sayı olup Şekil 4'deki kesikli çizgilerin aralığının boyutunu belirlemek için kullanılmaktadır. $C$ parametresinin belirlenmesi veri setine göre değişiklik göstermektedir. $\mathrm{Bu}$ yüzden deneme yanılma yoluyla tespit edilmektedir. $\xi_{i}$ ve $\xi_{i}^{*}$ ise aykırı gözlemlerin kesikli çizgiye olan mesafelerini belirtmektedir. $\varepsilon$ sıfırdan büyük bir sayı olup kullanıcı tarafindan belirlenmektedir. Eşitlik (53)' deki $b$ katsayısı oluşturulan fonksiyonda algoritmanın belirlediği bir sabit sayıdır. $w$ ise verilen $x i$ giriş değişkenleri ile çarpılacak sayı olup algoritma tarafindan belirlenmektedir. Şekil 5'te görüldüğü gibi UKF kestirimlerini iyileştirmek için SVM algoritmasının eğitimi, UKF kestiriminden elde edilen veriler ile yapılmıştır. SVM eğitiminde kullanılan veri seti 3 adet sigma noktas1, UKF kestirimi ve insansız sualtı aracının matematiksel modelinden alınan gerçek değer olmak üzeri her bir örnekleme aralığı için 5 farklı veriden oluşmaktadır.

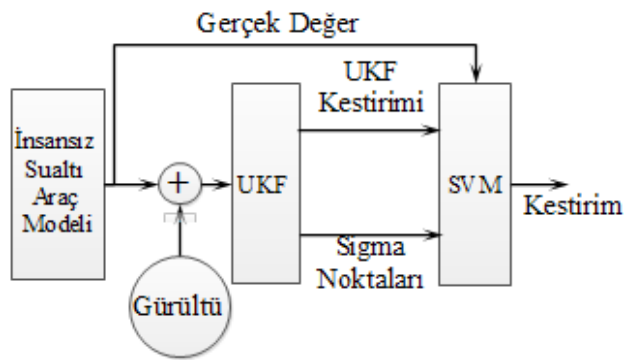

Şekil 5. SVM/UKF blok şeması

Şekil 6'da gösterildiği gibi EKF kestirimlerini iyileştirmek için ise SVM algoritmasının eğitimi, EKF kestiriminden elde edilen veriler ile yapılmıştır. SVM eğitiminde kullanılan veri seti, EKF kestirimi ve insansız sualtı aracının matematiksel modelinden alınan gerçek değer olmak üzeri her bir örnekleme aralığı için 2 farklı veriden oluşmaktadır.

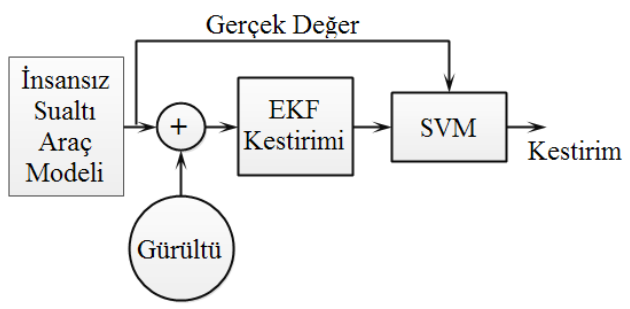

Şekil 6. SVM/EKF blok şeması

\section{SIMUÜLASYON SONUÇLARI}

Bu çalışmada kullanılan insansız sualtı aracının sağ ve sol iticilerine eşit kuvvet uygulayarak ileri yön hareketi analizi, sağ ve sol iticilere farklı kuvvet uygulayarak ise sapma açısı (yaw) analiz edilmiştir. Buna ek olarak, insansız sualtı aracının belirtilen kare bir yolu takip etmesi sağlanmıştır ve bu kare yol takip hareketi için, oluşturulan filtrelerin başarımları karşılaştırılmıştır.

$\mathrm{Bu}$ çalışmada insansız sualtı aracının farklı gürültülerdeki konum ve yönelim hareketi incelenmiştir. Şekil 7 ve Şekil 9'da farklı gürültülerde aracın ileri yön hareketi için, matematiksel model cevabından elde edilen $\mathrm{x}$ eksenindeki gerçek konum bilgisi ile EKF (sarı çizgi) ve UKF (mavi çizgi) ile SVM (kırmızı ve yeşil çizgi) algoritmalardan elde edilen konum kestirimi gösterilmiştir. Şekil 8 ve Şekil 10 'da x konum kestirimindeki elde edilen mutlak hata grafikleri UKF (mavi çizgi), EKF (sarı çizgi), SVM/UKF (kırmızı çizgi) ve SVM/EKF (yeşil çizgi) için verilmiştir. Mutlak hata kestirilen veri ile aracın model cevabından elde edilen gerçek veri arasındaki fark olarak tanımlanmıştır. Şekil 8 ve $10^{\prime}$ da görüldüğü gibi az gürültülü ve çok gürültülü durumda en düşük hata SVM/UKF algoritması ile elde edilmiştir.

Şekil 11 ve Şekil 13'de farklı gürültülerde aracın sapma yön hareketi için, matematiksel model cevabından elde edilen sapma açısı (siyah çizgi) ile EKF (sarı çizgi) ve UKF (mavi çizgi) ile SVM algoritmalardan (kırmızı, yeşil çizgi) elde edilen sapma açısı kestirimleri sunulmuştur. Şekil 12 ve Şekil 14'te sapma açısı kestiriminde elde edilen mutlak hata grafiği verilmiştir. Sapma yönelim hareketi için, az gürültülü ve çok gürültülü durumlarda SVM/UKF algoritmanın en düşük hataya sahip olduğu Şekil 12 ve 14 'te görülmektedir.

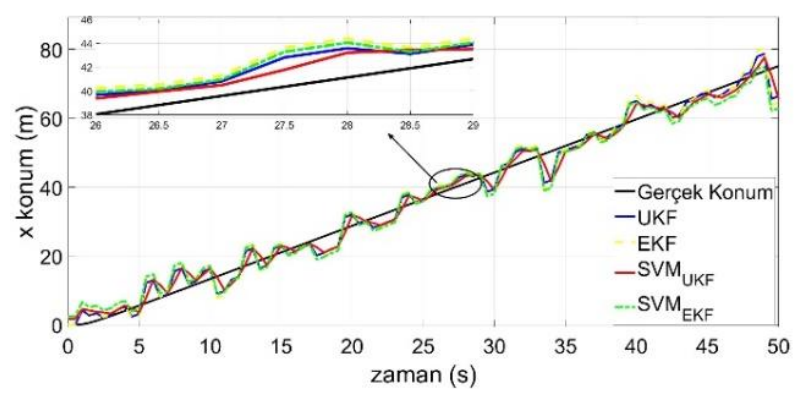

Şekil 7. Zamana bağlı gerçek x konumu ve kestirimleri (az gürültülü durum) 


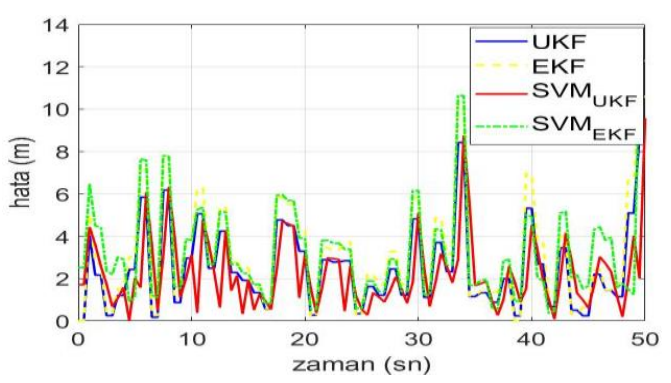

Şekil 8. x konum kestirimi için mutlak hata (az gürültülü durum)

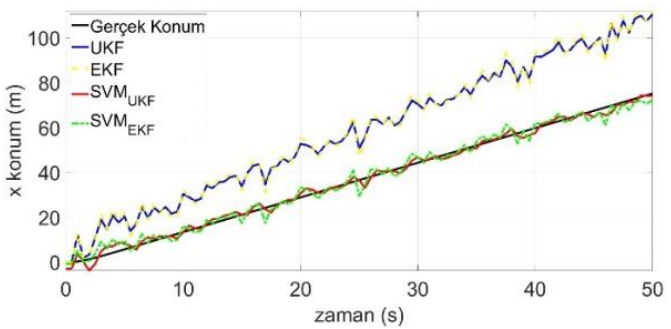

Şekil 9. Zamana bağlı gerçek x konumu ve kestirimleri (çok gürültülü durum)

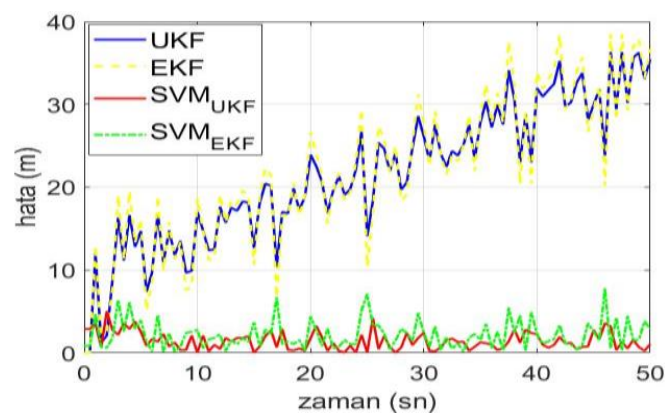

Şekil 10. $x$ konum kestirimi için mutlak hata (çok gürültülü durum)

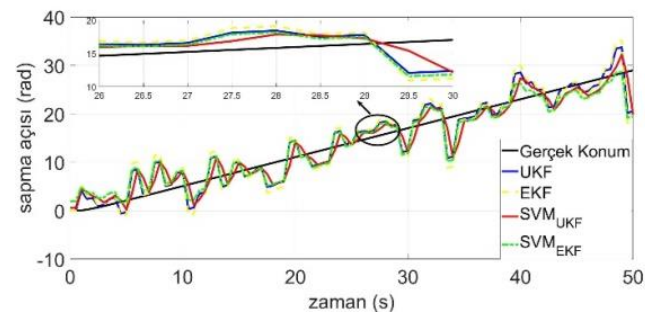

Şekil 11. Zamana bağlı sapma açısı ve kestirimleri (az gürültülü durum)

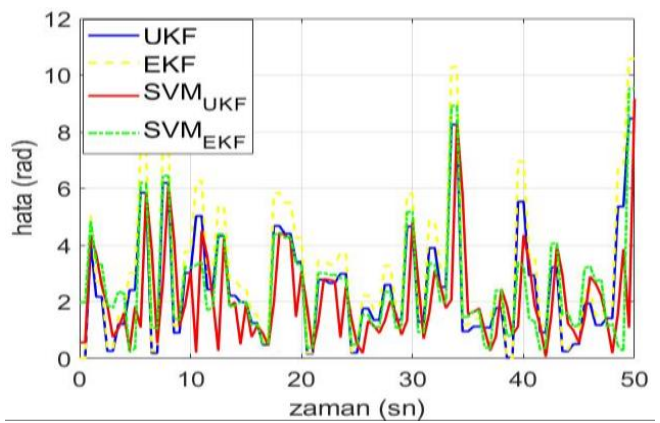

Şekil 12. Sapma açısı kestirimi için mutlak hata (az gürültülü durum)

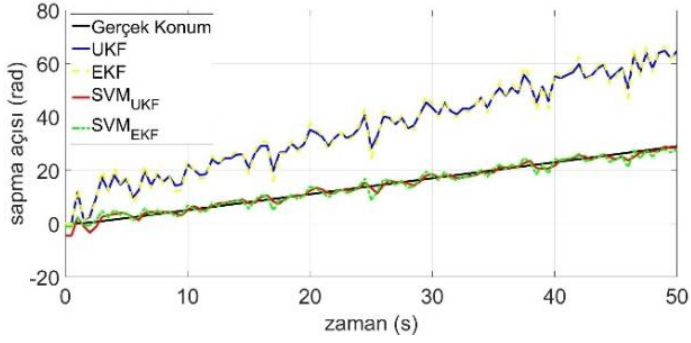

Şekil 13. Zamana bağlı gerçek sapma açısı ve kestirimleri (çok gürültülü durum)

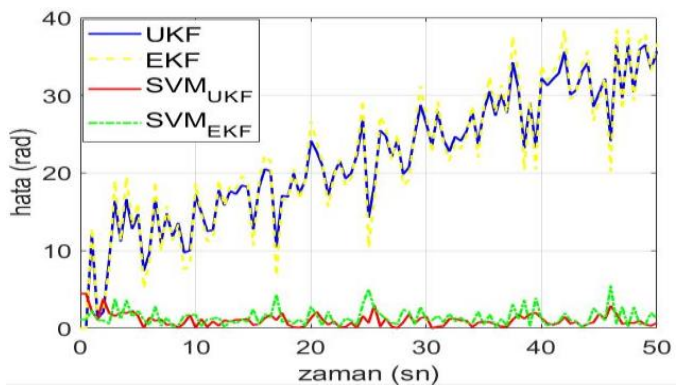

Şekil 14. Sapma açısı kestirimi için mutlak hata (çok gürültülü durum)

$\mathrm{Az}$ gürültülü ve çok gürültülü ortamlarda her bir algoritmanın mutlak hatası analiz edilmiştir. Mutlak hata her bir algoritma tarafindan kestirilen veri ile aracın model cevabından üretilen gerçek veri arasındaki farktır. Tablo 1 de, az gürültülü ve çok gürültülü durumlarda $\mathrm{EKF}, \mathrm{UKF}, \mathrm{SVM} / \mathrm{UKF}$ ve $\mathrm{SVM} / \mathrm{EKF}$ algoritmaları ile aracin kestirilen $\mathrm{x}$ konumu ve gerçek $\mathrm{x}$ konumu arasındaki mutlak hata verilmiştir. Buna ek olarak, EKF, UKF, SVM/UKF ve $S V M / E K F$ algoritmaları ile aracin kestirilen sapma açısı ve gerçek sapma açısı arasındaki fark (mutlak hata) verilmiştir. Tablo 1'dende görüldüğü gibi hem az gürültülü hem de çok gürültülü durumda en doğru sonuç SVM algoritmasının UKF ye entegre edildiği durumdur.

Tablo 1. Filtre kestirimler için mutlak hata değerleri

\begin{tabular}{|l|l|l|l|}
\hline \multirow{2}{*}{ ALGORITMA } & \multicolumn{3}{|l|}{ MUTLAK HATA } \\
\cline { 2 - 4 } & $\mathbf{x}$-Konum & $\mathbf{y}$-Konum & $\begin{array}{l}\text { Sapma } \\
\text { Açisı }\end{array}$ \\
\hline SVM UKF & $1,529 \mathrm{~m}$ & $2,0977 \mathrm{~m}$ & $0,1254 \mathrm{rad}$ \\
\hline SVMEKF & $2,6819 \mathrm{~m}$ & $2,3262 \mathrm{~m}$ & $0,1509 \mathrm{rad}$ \\
\hline
\end{tabular}

$\mathrm{Bu}$ çalışmada SVM ile iyileştirilmiş filtrelerin başarımları $\mathrm{x}$ ve $\mathrm{y}$ eksenlerinde $75 \mathrm{~m} \times 75 \mathrm{~m}$ olarak verilen kare yol hareketi için karşılaştırılmıştır. Aracın verilen kare yolu takip edebilmesi kontrolcüler ile uygun girdi bilgileri üretilerek sağlanmıştır. Verilen kare yol için iyileştirilmiş filtre kestirimleri Şekil 15'te verilmiştir. Şekil 16 ve 17 'de sırasıyla x eksninde alınan yol ile y ekseninde alınan yola ait mutlak hatalar verilmiştir. Sapma açısı için iyileştirilmiş filtre kestirimleri Şekil 18 verilmiştir. Sapma açısına ait mutlak hatalar SVM/UKF ve SVM/EKF için Şekil 19'da verilmiştir. 


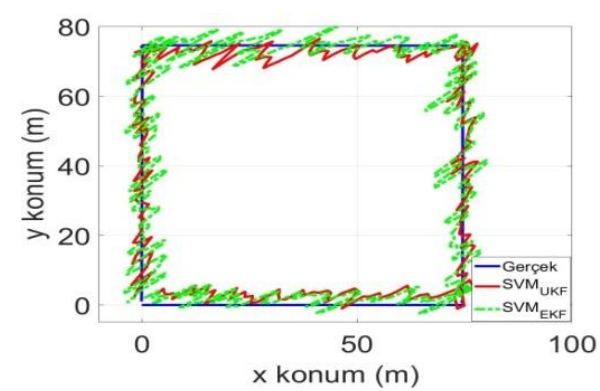

Şekil 15. Kare yol için filtre kestirimleri

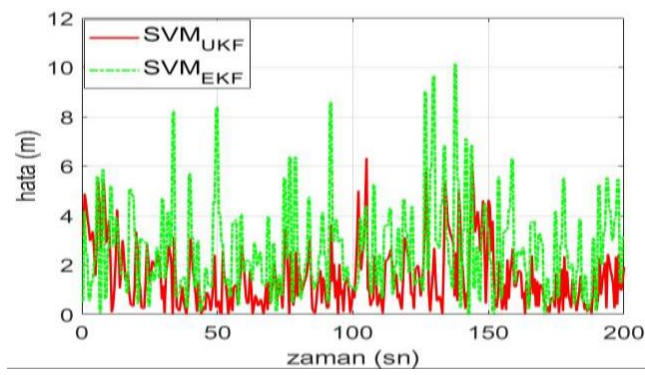

Şekil 16. Kare yol için $\mathrm{x}$ ekseninde alınan yol (x) için mutlak hata

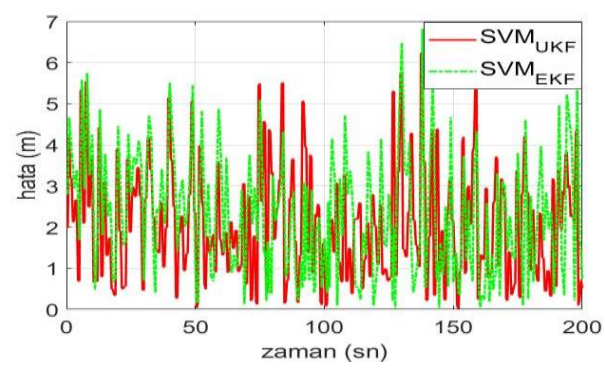

Şekil 17. Kare yol için y ekseninde alınan yol (y) için mutlak hata

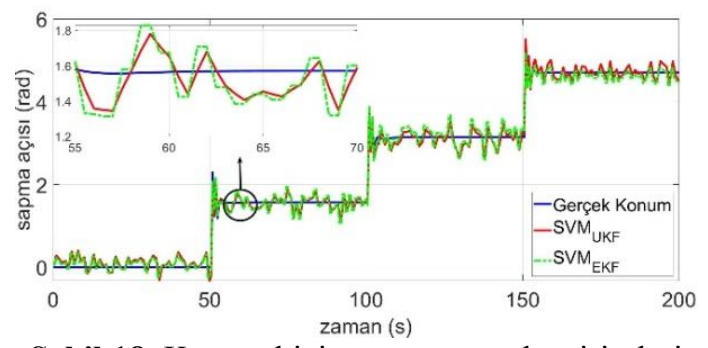

Şekil 18. Kare yol için sapma açısı kestirimleri

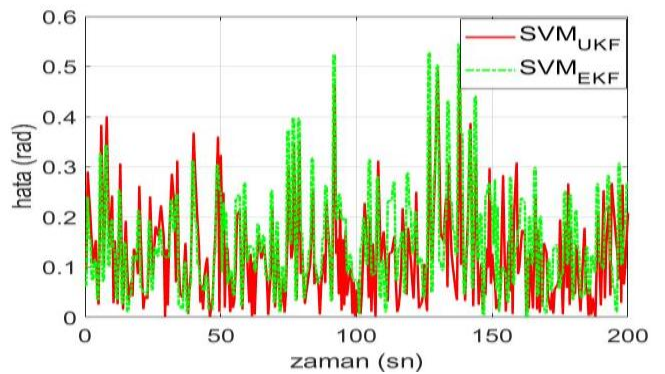

Şekil 19. Kare yol için sapma açısının mutlak hatası
Tablo 2 de aracın takip ettiği kare bir yola ait SVM/UKF ve SVM/EKF algoritmalarına kestirilen konum $(\mathrm{x}, \mathrm{y})$ ve sapma açısına ait mutlak hatalar verilmiş̧ir. Mutlak hata kestirilen veri ile aracın model cevabından elde edilen gerçek veri arasındaki farktır. Tablo 2'den de görüldüğü gibi SVM/UKF'nin doğruluğu SVM/EKF’ye göre daha büyüktür.

Tablo 2. Kare yol kestirimi için mutlak hata değerleri

\begin{tabular}{|l|l|l|l|}
\hline \multirow{2}{*}{ ALGORITMA } & \multicolumn{3}{|l|}{ MUTLAK HATA } \\
\cline { 2 - 4 } & $\begin{array}{l}\text { konum } \\
(\mathbf{x})\end{array}$ & $\begin{array}{l}\text { konum } \\
(\mathbf{y})\end{array}$ & $\begin{array}{l}\text { sapma } \\
\text { açısı }\end{array}$ \\
\hline SVMUKF & $1,529 \mathrm{~m}$ & $2,0977 \mathrm{~m}$ & $0,1254 \mathrm{rad}$ \\
\hline SVM $_{\mathrm{EKF}}$ & $2,6819 \mathrm{~m}$ & $2,3262 \mathrm{~m}$ & 0,1509 \\
\hline
\end{tabular}

\section{V.SONUC}

Matematiksel modeli elde edilen insansız sualtı aracının gerçek konum ve yönelim bilgilerine gürültü eklenerek navigasyon sensör verisi üretilmiştir. $\mathrm{Bu}$ sensör verilerinden konum ve sapma açısı kestirimi için UKF, EKF algoritmaları uygulanmıştır. Sonuçlardan UKF'nin kestirim performansı daha iyi olduğu görülmektedir. Çok gürültülü ortamlardaki UKF ve EKF kestirimlerini iyileștirmek için farklı makine öğrenmesi algoritmaları test edilmiştir. Test edilen algoritmalardan en iyi sonucu veren SVM algoritması UKF ve EKF kestirimlerine uygulanmıştır. İyileştirilmiş filtre kestirimlerinden başarımı en yüksek olan SVM $_{\mathrm{UKF}}$ (SVM algoritması ile iyileştirilmiş UKF) algoritması olduğu gözlemlenmiştir. Bunun nedeni, SVM algoritmasının veri setini oluşturmak için veri çeşitliliği UKF algoritmasinda mevcuttur. SVM veri setini UKF algoritması ile oluştururken UKF kestirimi ve sigma noktaları kullanılmıștır. Ancak SVM veri setini EKF ile oluştururken sadece EKF filtre kestirimi kullanılabilir. Bu nedenle çok gürültülü ortamlarda bile SVM $_{\mathrm{UKF}}$ algoritması gerçeğe yakın konum ve yönelim kestirimi yapabilmektedir. İyileştirilmiş filtre başarımlarını daha iyi karşılaştırmak için insansız sualtı aracının verilen kare bir yolu takip etmesi sağlanmıştır. Verilen bu kare yolda da SVM UKF $_{\text {f }}$ algoritmasının gerçeğe daha yakın olduğu görülmektedir. Tüm çalışma MATLAB/Simulink ortamında gerçekleştirilmiştir.

Gelecek çalışmada, sualtı aracı ile denizde gerçek navigasyon deneyleri yapılarak alınan ölçümlere bu çalışmadaki algoritmalar uygulanarak karşılaştırılacaktır.

\section{TEŞEKKÜR}

Bu çalıșma 119E037 nolu TÜBİTAK 1001 projesi dâhilinde desteklenmiştir.

$\mathrm{Bu}$ yayının bir kısmı, genişletilmemiş kısmı ASYU 2020 konferansında sözlü olarak sunulmuştur. 


\section{KAYNAKLAR}

[1] Groves, P. D. (2013). Principles of GNSS, Inertial, and Multisensor Integrated Navigation Systems., Artech House.

[2] Liu , K.-Z., Li , J., Guo, W., Zhu , P.-q., ve Wang , X.-h.(2014) Navigation system of a class of underwater vehicle based on adaptive unscented Kalman fiter algorithm, Springer, 550-557.

[3] Duymaz, E., Oğuz, A. E., ve Temeltaş, H. (2017). Eş zamanlı konum belirleme ve haritalama probleminde yeni bir durum tahmin yöntemi olarak parçacık akış filtresi. DergiPark, 1255 1270.

[4] Jwo, D. J., Hu, C. W., ve Tseng, C. H. (2013). Nonlinear Filtering with IMM Algorithm for Ultra-Tight GPS/INS Integration. SAGE, 116.

[5] St-Pierre, M., Gingras, D. (2004). Comparison between the unscented Kalman filter and the extended Kalman filter for the position estimation module of an integrated navigation information system, Proc. of IEEE Intelligent Vehicles Symposium, 831-835.

[6] Makavita, C. D., Jayasinghe, G. S., Nguyen, D. H., ve Ranmuthugala, D. (2019). Experimental Study of Command Governor Adaptive Control for Unmanned Underwater Vehicles. IEEE, 332 - 345.

[7] Daum, F. (2005). Nonlinear filters: beyond the Kalman filter. IEEE, $57-69$.

[8] Wan, E. A., Merwe, R. V. (2000). The unscented Kalman filter for nonlinear estimation. Proceedings of the IEEE 2000 Adaptive Systems for Signal Processing, Communications, and Control Symposium, Lake Louise: IEEE, 153-158.

[9] Kandepu R., Foss, B., Imsland, L. (2008). Applying the unscented Kalman filter for nonlinear state estimation, J. Process Control, 753-768.

[10] Xiong, K., Zhang, H. Y. ve Chan, C. W. (2006). Performance evaluation of UKF-based nonlinear filtering. ELSEVIER, 261-270.

[11] Dini, D. H., Mandic, D. P. ve Julier, S. J. (2011). A Widely Linear Complex Unscented Kalman Filter. IEEE, 623 - 626.

[12] Menegaz, H. M. T., Ishihara, J. Y., Borges, G. A. ve Vargas, A. N. (2015). A Systematization of the unscented Kalman filter theory. IEEE Trans. Automat. Control, 60 (10), 2583-2598.

[13] Holmes, S. A., Klein, G. ve Murray, D. W. (2008) An O(N2) Square Root Unscented Kalman Filter for Visual Simultaneous Localization and Mapping[J]. IEEE Transactions on Pattern Analysis \& Machine Intelligence, 31 (7), 1251-1263.

[14] Huang, M., Li, W., Yan, W. (2010). Estimating parameters of synchronous generators using square-root unscented Kalman filter Electr. Power Syst. Res., 80 (9), 1137-1144

[15] Erol, B., Cantekin, R.F., Kartal, S.K., Hacioğlu, R., Görmüş, K.S., Kutoğlu, S.H. ve Leblebicioğlu, M.K. (2020). Improvement of filter estimates based on data from unmanned underwater vehicle with machine learning. Innovations in Intelligent Systems and Applications Conference (ASYU). Istanbul, Turkey. 15-17 October. IEEE.

[16] Solomatine, D. P., Shrestha, D. L. (2009). A novel method to estimate model uncertainty using machine learning techniques. Water Resources Research, 45(12), 1-16.

[17] Cortes, C., Vapnik, V. (1995). Support-vector networks Machine Learning, 273-297

[18] Zhang, Z., Ding, S., Sun, Y., (2021), MBSVR: Multiple birth support vector regression. Information Sciences, 552, 65-79.

[19] Zhao, Q., Qin, X., Zhao, H., ve Feng, W. (2018). A novel prediction method based on the support vector regression for the remaining useful life of lithium-ion batteries. Microelectronics Reliability, 85, 99-108.

[20] Li, X., Shu, X., Shen, J., Xiao, R., Yan, W., ve Chen, Z. (2017). An On-Board Remaining Useful Life Estimation Algorithm for Lithium-Ion Batteries of Electric Vehicles. Energies, 10(5), 691.

[21] Oktanisa, I., Mahmudy, W. F., ve Maski, G. (2020). Inflation Rate Prediction in Indonesia using Optimized Support Vector Regression Model. Journal of Information Technology and Computer Science, 5(1), 104-114.

[22] Manasa, J., Gupta, R., ve Narahari, N. S. (2020). Machine Learning based Predicting House Prices using Regression Techniques. 2020 2nd International Conference on Innovative Mechanisms for Industry Applications (ICIMIA), 624-630.

[23] Smola, A. J., Schölkopf, B. (2004). A tutorial on support vector regression. Statistics and Computing, 14(3), 199-222.

[24] Dong, Y., Zhang, Z., Hong, W.C. (2018). A hybrid seasonal mechanism with a chaotic cuckoo search algorithm with a support vector regression model for electric load forecasting. MDPI. 1-21.

[25] Li, M.W., Geng, J., Hong, W.C., Zhang, L.D. (2019). Periodogram estimation based on LSSVR-CCPSO compensation for forecasting ship motion. Nonlinear Dynamics, 97 (4), 2579-2594.

[26] Cheng, K., Lu, Z. (2021). Active learning Bayesian support vector regression model for global approximation. Information Sciences, 544, 549-563.

[27] Zhang, Z., Ding, S., Sun, Y. (2020). A support vector regression model hybridized with chaotic krill herd algorithm and empirical 
mode decomposition for regression task. Neurocomputing, 410, 185-201.

[28] Fossen, T. I. (1999) Guidance and Control of Ocean Vehicles. 1. bask1, Wiley.

[29] Kartal, S.K, Leblebicioğlu, M.K., ve Ege, E. (2016). Bir Ínsansız Sualtı Gözlem Aracı (SAGA) Yer Tespitinin Deneysel Testi ve Sistem Tanılaması. Otomatik Kontrol Ulusal Toplantisi (TOK), 1-13.

[30] Crassidis, J. L., Junkins, J. L. (2011). Optimal Estimation of Dynamic Systems (Chapman \& Hall/CRC Applied Mathematics \& Nonlinear Science). Chapman and Hall/CRC.
[31] Merwe, R. V., Wan, E. A. (2001). The squareroot unscented Kalman filter for state and parameter-estimation. $2001 \quad$ IEEE International Conference on coustics, Speech, and Signal Processing, Salt Lake City: IEEE, 3461-3464.

[32] Haykın, S. (2001). Kalman Filtering and Neural Networks, 1. Bask1. Wiley-Interscience, New York, USA.

[33] Vapnik, V. (2000). The Nature of Statistical Learning Theory. 2. bask1. Springer-V. 